\title{
AVALIAÇÃO IN VITRO DOS EFEITOS INSETICIDA E LARVICIDA DE OITO ÓLEOS ESSÊNCIAIS SOBRE O CASCUDINHO AVIÁRIO (Alphitobius diaperinus)
}

\author{
(In vitro evaluation of the insecticide and larvicide effects of eight essential oils on \\ mealworm (Alphitobius diaperinus))
}

Andreia Volpato ${ }^{1}$, Gabriela Mioto Galli ${ }^{1}$, Gabriela Campigotto ${ }^{1}$, Patricia Glombowsky ${ }^{1}$, Roberto Christ Vianna Santos ${ }^{2}$, Aleksandro Schafer da Silva ${ }^{1 *}$, Rodrigo de Almeida Vaucher ${ }^{3}$

\footnotetext{
${ }^{1}$ Universidade do Estado de Santa Catarina, Brasil. *Correspondência: aleksandro_ss@yahoo.com.br 2Universidade Federal de Santa Maria, Rio Grande do Sul, Brasil.

${ }^{3}$ Universidade Federal de Pelotas, Pelotas, Rio Grande do Sul, Brasil. Rio Grande do Sul, Brasil.
}

RESUMO: Alphitobius diaperinus, popularmente conhecido como cascudinho, é um inseto que se destaca na avicultura por ser vetor de patógenos e de difícil controle. Esta dificuldade está relacionada à contraindicação na utilização de produtos químicos, já que enquanto as aves estão no aviário, grandes prejuízos podem ocorrer em consequência de elevadas infestações. Portanto, produtos naturais com potencial inseticida e larvicida poderiam ser usados, sem prejudicar as aves e sem deixar resíduo na carne do frango. Em virtude disso, neste estudo objetivou-se verificar o efeito dos óleos essenciais de Capim limão (Cymbopogon citratus), Cedro (Cedrus atlantica), Gengibre (Zingiber officinale), Gerânio (Pelargonium graveolens), Junípero (Juniperus communis), Palmarosa (Cymbopogon martinii), Tomilho (Thymus vulgaris) e Vetiver (Vetiveria zizanioides) sobre a suscetibilidade de $A$. diaperinus. Os testes foram realizados em triplicata com os óleos essenciais nas concentrações de 1, 5 e $10 \%$. A fim de validar os testes, foram utilizados dois controles: sem tratamento e com diluente (Triton). Os efeitos larvicida e inseticida do óleo de Capim limão não foi satisfatório, pois a taxa de mortalidade superior a $50 \%$ foi atingida apenas 14 e 16 dias após o início do teste, respectivamente. O óleo de Cedro não demostrou efeito significativo sobre adultos e larvas, pois os valores de mortalidade foram inferiores a $50 \%$ em todas as concentrações avaliadas. Os efeitos inseticidas e larvicidas não foram observados nos demais óleos nas concentrações testadas neste experimento $(P>0,05)$. Portanto, com base nos resultados de baixa ação inseticida e larvicida dos óleos testados sobre $A$. diaperinus in vitro, concluímos que os mesmos são inadequados para o controle alternativo desta praga

Palavras-chave: controle alternativo; óleos essenciais; inseticida; avicultura

ABSTRACT: Alphitobius diaperinus, the aviary mealworm, is an insect that stands out in poultry to be vector of pathogens and difficult to control. The difficulty in control is related to the contraindication in the use of chemicals while the birds are in the aviary, and large losses can occur as a result of high infestations. Therefore, natural products with potential insecticide and larvicide could be used without harming the birds, as well as without leaving residue in chicken meat. As a result, this study aimed to verify the effect of Lemon Grass oil (Cymbopogon citratus), Cedar (Cedrus atlantica), Ginger (Zingiber officinale), Geranium (Pelargonium graveolens), Junipero (Juniperus communis), Palmarosa (Cymbopogon martinii), Thyme (Thymus vulgaris) and Vetiver (Vetiveria zizanioides) on the susceptibility of 
A. diaperinus. The tests were performed in triplicate with essential oils at concentrations of 1,5 , and $10 \%$. In order to validate the tests two controls were used: untreated and diluent (Triton). The larvicide and insecticidal effects of lemon grass oil was not satisfactory because the mortality rate higher than $50 \%$ was reached only 14 and 16 days after start of the test, respectively. Cedar oil did not show significant effect on adults and larvae, as the mortality values were less than $50 \%$ at all concentrations tested. The effect of insecticide and larvacide were not observed in the other oils at concentrations tested in this experiment $(P>0.05)$. Therefore, based on the results of the in vitro insecticidal and larvicidal low-action tests of the oils tested on $A$. diaperinus, we conclude that they are inadequate for the alternative control of this pest.

Keywords: alternative control; natural products; insecticide; poultry

\section{INTRODUÇÃO}

A avicultura no Brasil é uma atividade agropecuária moderna e de alta produtividade, com destaque no mercado internacional. Este cenário promove a utilização intensiva dos recursos necessários para a produção, como a maravalha (cama). No entanto, este fator favorece o crescimento da população do Alphitobius diaperinus, conhecido popularmente como cascudinho (CHERNAKI-LEFFER et al., 2002). Este inseto é uma das principais pragas da avicultura moderna e habita o substrato utilizado no aviário, um ambiente com temperatura e umidade propícia para seu crescimento, além de alimentar-se de ração, excretas e aves mortas (ROHDE et al., 2006). Este coleóptero pode ser responsável pela baixa conversão alimentar das aves, visto que estas ingerem o cascudinho ao invés de uma alimentação equilibrada. Além disto, é um possível veiculador de diferentes patógenos para aves saudáveis, tais como fungos (Aspergillus sp.), bactérias (Escherichia sp., Salmonella sp., Bacillus sp., Streptococcus sp.), vírus (vírus da leucose aviária, vírus da Doença de Gumboro, Coronavirus) e parasitas (Eimeria sp., Choanotaenia sp. e Raillietina sp), causadores de problemas econômicos e sanitários, que afetam a saúde e o crescimento das aves, além de comprometer a segurança alimentar dos consumidores (DE LAS CASA et al., 1972; CHERNAKI-LEFFER et al., 2002).

Atualmente, as práticas de manejo adotadas para o controle são baseadas em produtos químicos aplicados na superfície da cama do aviário. Porém, embora sejam eficientes, causariam intoxicação nas aves. Além disso, o habito biológicos destes insetos também é outro complicador no controle, pois durante o dia permanecem em galerias e a noite migram para as asas das aves (ALVES et al., 2005). Além de todas estas dificuldades, alguns inseticidas sintéticos como cipermetrina e diclorvos tem registros de baixa eficácia contra o cascudinho (CHERNAKI-LEFFER et al., 2011).

$\begin{array}{ccc}\text { Nos } & \text { últimos } & \begin{array}{r}\text { anos, } \\ \text { pesquisadores }\end{array} \text { têm } \\ \text { estudado }\end{array}$ alternativas para 0 controle do $A$. diaperinus, como 0 uso de microrganismos entomopatogênicos no controle biológico, por serem inócuos aos animais endotérmicos, incluindo as aves (ALVES et al., 2005). A utilização de produtos naturais é outro método que tem se destacado no controle de 

cascudinho aviário (Alphitobius diaperinus)

diversos microrganismos patogênicos e pragas. Esses produtos podem ser uma alternativa eficiente e viável para o controle do cascudinho, em vista que este método não depende da ausência das aves nos aviários. Alguns óleos essenciais já foram testados e tiveram seu efeito inseticida comprovado, como os óleos de Cunila angustifolia (DO PRADO et al., 2013) e Melaleuca alternifolia (VOLPATO et al., 2016). Portanto, o objetivo deste estudo foi avaliar pela primeira vez a suscetibilidade in vitro de adultos e larvas de $A$. diaperinus frente a oito diferentes óleos essenciais, com diferentes propriedades medicinais já descritas na literatura.

\section{MATERIAL E MÉTODOS}

Os cascudinhos foram coletados de aviários naturalmente infestados localizados na região de Chapecó, estado de Santa Catarina, Brasil. Os adultos e larvas foram coletados manualmente, armazenados em frascos plásticos ventilados e transportados ao laboratório. Permaneceram nos frascos plásticos ventilados com substrato de aviário e algodão umedecido com água para proporcionar um ambiente favorável, recebendo ração de frango de corte como alimentação.

Os testes in vitro foram realizados em placas de Petri de $10 \mathrm{~cm}$ de diâmetro e $2 \mathrm{~cm}$ de altura. No interior das placas, a região do fundo foi revestida com papel filtro para aplicação e absorção de $300 \mu \mathrm{L}$ da solução testada, de modo que os indivíduos não tivessem contato direto com solução teste. Os óleos foram inicialmente diluídos em Triton na proporção 1:1, pois não são miscíveis com a água. Posteriormente, a solução foi diluída em água destilada nas concentrações finais de 1,5 e 10\%. Para validar os testes, utilizaram-se grupos controle com aplicação de Triton a 10\% (maior concentração de óleo testada) e grupo sem aplicação de qualquer tratamento (óleo ou diluente). Cada placa de Petri recebeu 10 adultos ou 10 larvas na fase L8 (última fase antes de pupa). Para cada tratamento foram realizadas três repetições em delineamento inteiramente casualizado.

Durante todo 0 período do experimento foi alocado diariamente na placa de Petri um algodão umedecido com água e ração (a base milho e farelo de soja). As placas de Petri ficaram alojadas em estufa climatizada à temperatura de $25 \pm 3^{\circ} \mathrm{C}$ e umidade relativa de $70 \pm 10 \%$, com fotoperíodo de 12 horas. O número de indivíduos vivos em cada parcela foi observado periodicamente.

Os óleos de Capim limão (Cymbopogon citratus), Cedro (Cedrus atlantica), Gengibre (Zingiber officinale), Gerânio (Pelargonium graveolens), Junípero (Juniperus communis), Palmarosa (Cymbopogon martinii), Tomilho (Thymus vulgaris) e Vetiver (Vetiveria zizanioides) usados nesse estudo foram comprados da empresa FERQUIMA ${ }^{\circledR}$ (Vargem Grande Paulista, São Paulo, SP, Brasil).

$O$ teste de normalidade foi aplicado nos bioensaios com cascudinho, que apresentou distribuição normal dos dados. Em seguida, os dados foram analisados estatisticamente por análise de variância e Teste de Duncan $(\mathrm{P}<0,05)$.

\section{RESULTADOS}

Os resultados da porcentagem de larvas vivas após os bioensaios com os óleos essenciais estão apresentados na Figura 1. Os testes tiveram duração média de 13 e 17 dias para larvas e adultos, respectivamente. $\mathrm{O}$ efeito larvicida do óleo de Capim limão não foi 
satisfatório nas concentrações testadas, pois as taxas de mortalidade são baixas para o tratamento ser considerado eficaz, apesar de apresentar os melhores resultados em relação aos demais óleos do experimento e diferença estatística significativa em relação ao controle $(P>0,05)$ (Fig. 1. A).

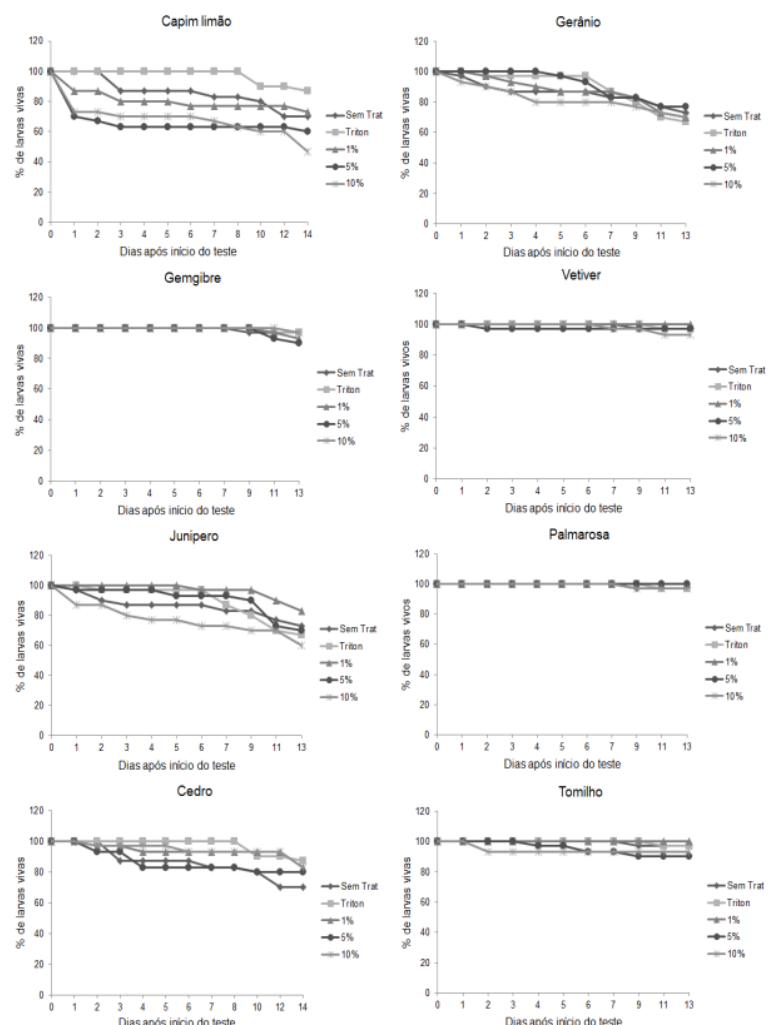

Figura 1 - Porcentagem de larvas vivas de Alphitobius diaperinus submetidas a testes nas concentrações 1, 5 e 10\% dos óleos essenciais de Capim limão (Cymbopogon citratus) (A), Gerânio (Pelargonium graveolens) (B), Gengibre (Zingiber officinale) (C), Vetiver (Vetiveria zizanioides) (D), Junípero (J. uniperus communis) (E), Palmarosa (Cymbopogon martinii) (F), Cedro (Cedrus atlantica) (G) e Tomilho (Thymus vulgaris) $(\mathrm{H})$.

Na maior concentração testada, a mortalidade superior a $50 \%$ foi atingida tardiamente, ou seja, aos 14 dias após aplicação do óleo. Sobre as larvas, as maiores percentagens de mortalidade foram vistas para os óleos de Junípero (40\%) e Gerânio (30\%) na concentração de $10 \%$ (Fig. 1. B e E). No entanto, essas porcentagens não são expressivas e 0 tratamento na concentração de $10 \%$ não diferiu estatisticamente dos grupos controle.

O óleo de Cedro apresentou mortalidade sobre larvas inferior a $20 \%$ na maior concentração testada, apenas 14 dias após o início do teste, mas essa porcentagem não é significativa em relação aos grupos controle (Fig. 1. G). Os óleos de Gengibre, Vetiver, Palmarosa e Tomilho não demostraram efeito sobre a mortalidade das larvas, pois os valores foram inferiores a $10 \%$ e não diferiram dos demais tratamentos (Fig. 1 - C, D, F e H).

As porcentagens de adultos vivos após os testes com os óleos essenciais foram apresentadas na Figura 2. O óleo de Capim limão apresentou o melhor efeito inseticida entre os óleos testados (Fig. 2. A). A mortalidade de $67 \%$ sobre os adultos na concentração de $10 \%$ apresentou diferença estatística significativa em relação aos demais tratamentos. No entanto, este valor foi atingido apenas 18 dias após aplicação do óleo, o que não é aceitável.

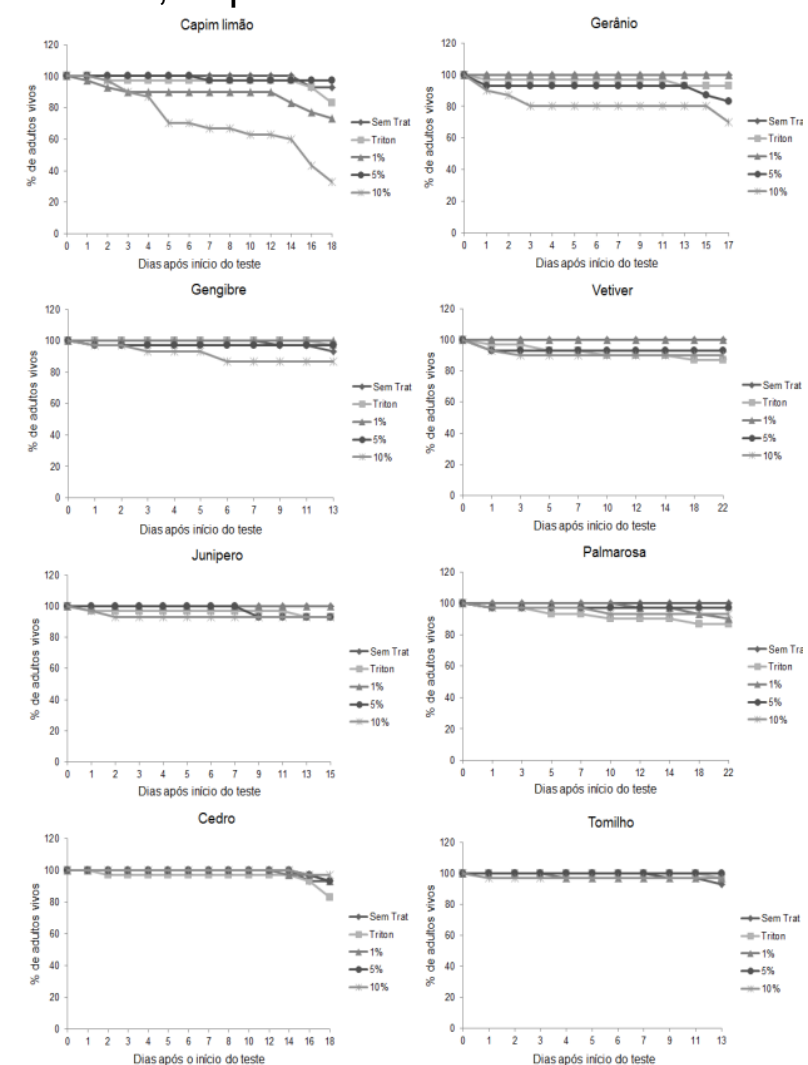

Figura 2- Porcentagem de adultos vivos de Alphitobius. diaperinus submetidos a testes nas 

cascudinho aviário (Alphitobius diaperinus)

concentrações 1, 5 e 10\% dos óleos essenciais de Capim limão (Cymbopogon flexuosus) (A), Gerânio (Pelargonium graveolens) (B), Gengibre (Zingiber officinale) (C), Vetiver (Vetiveria zizanioides) (D), Junípero (Juniperus communis) (E), Palmarosa (Cymbopogon martinii) (F), Cedro (Cedrus atlantica) (G) e Tomilho (Thymus vulgaris) $(\mathrm{H})$.

Com apenas $30 \%$ de mortalidade, 0 óleo de Gerânio teve baixa eficácia sobre adultos na concentração de $10 \%$ (Fig. 2. B). Os óleos essências de Gengibre, Vetiver, Junípero, Palmarosa, Cedro e Tomilho não apresentaram efeito inseticida em todas as concentrações avaliadas, pois os valores de mortalidade foram inferiores a $15 \%$ e não diferiram dos grupos controle (Fig. 2. C, D, E, F, G e H).

\section{DISCUSSÃO}

O manejo padrão adotado no controle de cascudinhos nos aviários é utilizado produtos químicos de forma intensiva, porém os riscos para a saúde, relacionados ao aplicador e às aves, e o impacto ao meio ambiente, estimulam a procura por controles alternativos que sejam eficientes e viáveis, além de oferecerem menor resistência parasitária, menor impacto ambiental e terem custo acessível. A utilização de produtos naturais, como os óleos essenciais, pode atender esses requisitos e por esses motivos vêm chamando a atenção dos pesquisadores. Portanto, o presente trabalho mostra 0 efeito de oito diferentes óleos essenciais sobre $A$. diaperinus. Até o presente momento, pesquisas com os óleos de C. flexuosus, C. atlantica, Z. officinale, P. graveolens, J. communis, C. martinii e V. zizanioides sobre cascudinhos não foram localizados pelo nosso grupo de pesquisa.

Foi verificado no presente trabalho que o óleo de Capim limão resultou na mortalidade de $57 \%$ dos adultos de $A$. diaperinus 16 dias após aplicação. Agnolin et al. (2013), verificaram importante ação acaricida do Capim limão sobre o carrapato Rhipicephalus microplus, ectoparasita bovino de grande importância sanitária. O efeito acaricida foi atribuído ao citral, seu composto majoritário, que também pode ter atividade sobre $A$. diaperinus.

No presente estudo, a aplicação do óleo essencial de Tomilho (T. vulgaris) não foi eficaz no controle de larvas e adultos. Assim como, no trabalho realizado por Szczepanik et al. (2012), a aplicação de $1 \%$ causou $17,5 \%$ de mortalidade das larvas velhas, mesmo estágio larval utilizado neste trabalho (SZCZEPANIK et al., 2012). Ambos os trabalhos mostram que o óleo essencial de Tomilho não é eficaz no controle de larvas de $A$. diaperinus, embora seu efeito esteja relacionado com a concentração e estágio larval. Atualmente é possível encontrar trabalhos mostrando os efeitos tóxicos de alguns dos óleos testados neste estudo sobre diversas espécies de parasitos, no entanto não existem relatos do efeito sobre $A$. diaperinus.

$O$ óleo de Gengibre resultou na mortalidade de $95 \%$ dos adultos de gorgulho-de-arroz (Sitophilus oryzae) (Linnaeus) (Coleoptera: Curculionidae), quando realizado aplicação tópica na dose de $20 \%$ (FRANZ et al., 2011). No mesmo trabalho, a dose de $10 \%$ resultou em $30 \%$ de mortalidade, taxa insatisfatória, ou seja, o óleo de gengibre não é eficaz em baixas concentrações (FRANZ et al., 2011). Assim como, o óleo essencial de Palmarosa no controle do caruncho (Callosobruchus maculatus) (Fabr.) (Coleoptera: Bruchidae), pois resultou em $100 \%$ de mortalidade nas concentrações testadas $(0,05 ; 0,10$; 0,$15 ; 0,20$ e $0,25 \%$ ) (PERREIRA et al., 2008). Apesar desse inseto ser da ordem do $A$. diaperinus, os resultados 
são negativos no presente estudo, o que pode estar relacionado a diversos fatores como por exemplo a resistência individual de cada inseto, composição do óleo que poderia varia entre regiões, países e períodos de coletas.

Testes com mosquito Anopheles atroparvus demostraram atividade inseticida do óleo de Junípero (ROCHA et al., 2008). O efeito inseticida também foi observado para Spodoptera frugiperda (lagarta-do-cartucho do milho) utilizando o óleo de Gerânio à 19,2\% (NICULAU et al., 2013). Da mesma forma, o estudo realizado com Coptotermes formosanus (cupim) demostrou que o óleo de Vetiver é eficaz como inseticida para esta espécie (MAISTRELLO e HENDERSON, 2000).

Com base nos resultados dos testes in vitro apresentados acima é possível concluir que os óleos testados neste estudo apresentam baixa ação inseticida e larvicida nas concentrações testadas sobre A. diaperinus, e, portanto, não são indicados no controle alternativo desta praga.

\section{REFERÊNCIAS}

AGNOLIN, C.A.; OLIVO, C.J.; PARRA, C.L.C. Efeito do óleo de capim limão (Cymbopogon flexuosus Stapf) no controle do carrapato dos bovinos. Revista Brasileira de Plantas Medicinais, v.16, n.1, p.77-82, 2014.

ALVES, L.F.A.; GASSEN, M.H.; PINTO, F.G.S. et al. Ocorrência natural de Beauveria bassiana (Bals.) Vuilleman (Moniliales: Moniliaceae) sobre 0 cascudinho, Alphitobius diaperinus (Panzer) (Coleoptera: Tenebrionidae), em aviário comercial de Cascavel, PR. Neotropical Entomology, v.34, n.3, p.507-510, 2005.

CHERNAKI-LEFFER, A.M.; BIESDORF, S.M.; ALMEIDA, L.M. et al. Isolamento de enterobactérias em Alphitobius diaperinus e na cama de aviários no Oeste do Estado do Paraná, Brasil. Revista Brasileira de Ciência Avícola, v.4, n.3, p.243-247, 2002.

CHERNAKI-LEFFER, A.M.; SOSAGÓMEZ, D.R.; ALMEIDA, L.M. et al. Susceptibility of Alphitobius diaperinus (Panzer) (Coleoptera, Tenebrionidae). Revista Brasileira de Entomologia, v.220, n.1, p.125-128, 2011.

DE LAS CASA, E.; HAREIN, P.K; POMEROY, B.S. Bacteria and fungi within the lesser mealworm collected from poultry brooder houses. Environmental Entomology, v.1, n.1, p.27-30, 1972.

FRANZ, A.R.; KNAAK, N.; FIUZA, L.M. Toxic effects of essential plant oils in adult Sitophilus oryzae (Linnaeus) (Coleoptera, Curculionidae). Revista Brasileira de Entomologia, v.55, n.1, p.116-120, 2011.

MAISTRELLO, L.; HENDERSON, G. Vetiver grass: useful tools against Formosan subterranean termites can be found in nature. Vetiver Newsletter, n.22, p.16-17, 2000.

NICULAU, E.S.; ALVES, P.B.; NOGUEIRA; P.C.L. et al. Atividade inseticida de óleos essenciais de Pelargonium graveolens l'Herit e Lippia alba (Mill) N. E. brown sobre Spodoptera frugiperda (J. E. Smith). Química Nova, v.36, n.9, p.1391-1394, 2013.

PEREIRA, A.C.R.L.; OLIVEIRA, J.V.; GONDIM JUNIOR, M.G.C. et al. Atividade inseticida de óleos essenciais e fixos sobre Callosobruchus maculatus (FABR., 1775) (Coleoptera: Bruchidae) em grãos de caupi [Vigna unguiculata (L.) WALP.]. Ciência e Agrotecnologia, v.32, n.3, p.717-724, 2008. 
DO PRADO, G.P.; STEFANI, L.M.; DA SILVA, A.S. et al. Alphitobius diaperinus (Coleoptera: Tenebrionidae) susceptibility to Cunila angustifolia essential oil. Journal of Medical Entomology, v.50, n.5, p.1040-1045, 2013.

ROCHA, D.K.; GRÁCIO, A.S.; MATOS, O.C. Importância das plantas aromáticas medicinais nas novas estratégias de controlo de vectores da malária. In: WORKSHOP PLANTAS MEDICINAIS E FITOTERAPÊUTICAS NOS TRÓPICOS, 2008, Lisboa. Anais... Lisboa: Instituto de Investigação Científica Tropical (IICT)/Centro Científico e Cultural de Macau (CCCM), 2008. p.1-10.

ROHDE, C.; ALVES, L.F.A.; NEVES, P.M.O.J. et al. Seleção de isolados de Beauveria bassiana (Bals.) Vuill. e Metarhizium anisopliae (Metsch.) sorok contra o cascudinho Alphitobius diaperinus (Panzer) (Coleoptera: Tenebrionidae). Neotropical Entomology, v.35, n.2, p.231-240, 2006.

SZCZEPANIK, M.; ZAWITOWSKA, B.; SZUMNY, A. Insecticidal activities of Thymus vulgaris essential oil and its components (thymol and carvacrol) against larvae of lesser mealworm, Alphitobius diaperinus Panzer (Coleoptera: Tenebrionidae). Allelopathy Journal, v.30, n.1, p.129142, 2012.

VOLPATO, A.; LORENZETTI, W. R.; ZORTEA, T. et al. Melaleuca alternifolia essential oil against the lesser mealworm (Alphitobius diaperinus) and its possible effect on the soil fauna. Brazilian Journal of Poultry Science, v.18, n.1, p.041-046, 2016. 